\title{
Microwave Photonics Analog Link based on two Integrated D-DPMZM Linearized Signals and with Eliminated odd Harmonics and all IMD's.
}

Shyqyri Haxha ( $\sim$ Shyqyri.Haxha@rhul.ac.uk)

Royal Holloway University of London

Shemsi shaqiri

Royal Holloway University of London

lan Flint

Royal Holloway University of London

Huseyin Ademgil

European University Cyprus

\section{Research Article}

Keywords: Optic modulators, Harmonic Distortions, Intermodulation distortion, Microwave Photonics

Posted Date: April 19th, 2021

DOI: https://doi.org/10.21203/rs.3.rs-419007/v1

License: (a) (i) This work is licensed under a Creative Commons Attribution 4.0 International License. Read Full License 


\title{
Microwave Photonics Analog Link based on two Integrated D-DPMZM Linearized Signals and with Eliminated odd Harmonics and all IMD's
}

\author{
${ }^{1}$ Shemsi ShaqIRI, ${ }^{*}$ Shyoyri HaXha, ${ }^{1}$ IAN Flint AND \\ ${ }^{2}$ HuSEYIN ADEMGIL \\ ${ }^{1}$ Department of Electronic Engineering School of Engineering, Physical and Mathematical Sciences \\ Royal Holloway, University of London, Egham, Surrey, TW20 OEX, United Kingdom. \\ ${ }^{2}$ Department of Computer Engineering, European University of Lefke, Lefke, Northern Cyprus, TR-10 \\ Mersin, Turkey.*Contact email:shyqyri.haxha@rhul.ac.uk
}

\begin{abstract}
A novel Microwave Photonic Link (MPL) system configuration based on two Dualdrive Dual-parallel Mach-Zehnder modulators (D-DPMZMs) and two Balanced PhotoDetectors (BPDs) is reported. The Intermodulation Distortions (IMDs), as well as the harmonic distortions have been eliminated. The proposed linearization of RF signal configuration is double side banded in both D-DPMZM. A full mathematical model has been developed and simulations have been implemented for the proposed configuration. The RF linearization for this configuration has been tested by introducing an additional RF signal, as well as phase and intensity errors in MZ branches. The proposed MPL system configuration exhibits significant performance and it will have great impact on aerospace, radar, and satellite-to-ground downlink communication system applications.
\end{abstract}

Index Terms: Optic modulators, Harmonic Distortions, Intermodulation distortion, Microwave Photonics

\section{Introduction}

Fiber optics communication is considered to be one of the most innovative methods of communication which has originated about 40 years ago. Radio-over-fiber (RoF) was introduced for the very first time in 1990 and since then it has been widely used whilst showing a great approach to communication system due to its high bandwidth, low losses, low power consummation, cost-effective and other of fiber-optic signal propagation properties [1-3]. RoF as an analogue communications system includes RF and optical fiber components such as: Photodiode, filters, optical amplifiers, optical fiber, optical modulator, and laser. So far optical communication system has resulted to be the best solution to modern mode communication due to above stated properties; however, optical communication has its own limitations as well, including non-linearity as a feature that produces Intermodulation Distortions (IMDs), loses and dispersion of signal. One of the most challenging tasks is the suppressing of nonlinear distortions, which strongly affects the performance of the Microwave Photonic Links (MPL).

Dual-parallel Mach-Zehnder modulator (DMZM) is most commonly used modulator for elimination or suppression of IMDs and possibly for dynamic increase range which requires complex radio-frequency arrangement and linearization $[4,6]$. Whereas Third Intermodulation Distortion (IMD3) is the most rigorous in signal linearization, down to being very close to transmitted signal and limiting free dynamic range. Conversely, when signals are very close to each other, the Second Intermodulation Distortions (IMD2) along Second Order Harmonics $(\mathrm{SOH})$ will experience severe distortions, subsequently many linearization techniques have been developed to supressing IMDs and improving Spurious-Free Dynamic Range (SFDR) [612].

In [13] improved linearized Analog Microwave Photonic Link (AMPL) with double dualparallel Mach-Zehnder modulator and a differential balanced photodetector is reported. In this paper, polarization maintained (PM) based optical components are used for better system stability. The developed theoretical model of the proposed system illustrates the elimination of even-order distortions and a high suppression to the IMD3 at the BPD. Consequently, the fundamental Signal to Interference ratio (S/I) of $60 \mathrm{~dB}$ was experimentally achieved. Experimental results, simultaneously, demonstrate a significant increase of Second-order Spurious free Dynamic Range (SFDR2) and Third-order Spurious-free Dynamic Range (SFDR3) by $19.5 \mathrm{~dB}$ and $3.1 \mathrm{~dB}$, respectively. The performance analysis of microwave photonic frequency conversion has been recorded to use a double-sideband suppressed-carrier and balance based on DPMZM [14]. The double-sideband technique has been used to suppress high harmonics and high Intermodulation's, as well as achieve frequency conversion signals. However, as suggested from the title, in [14], only manage to suppress theoretically IMDs and high harmonics.

Based on Dual-parallel Mach-Zehnder modulator (DPMZM), a linearization AMPL with IMD3 elimination is proposed and experimentally demonstrated [15]. Using symmetrical side band modulation, in this paper, the authors have managed to eliminate IMD3, in theory, by using two shifters and $3 \mathrm{~dB}$ power combiners. It has also been demonstrated experimentally the $45 \mathrm{~dB}$ suppression of the IMD3. Nevertheless, this paper only reports the elimination of IMD3 with 
all the other Harmonics and IMD's that has been left; consequently, in our paper we have used similar technique, whereas, we have managed to remove all IMD's. A dual-wavelength linearization of analogue photonic link, based on PM-IM conversion, has been proposed and demonstrated in [16]. Furthermore, a phase modulator which exhibits different electro-optic modulation index was used. Primarily, this paper reports and experimentally demonstrate a suppression of IMD3 by $14.54 \mathrm{~dB}$ based on two different channels with opposite field which than fabricate a possible suppression of IMD3.

Multi-octave linearized analogue photonic link based on a polarization DPMZM is proposed in [17]. An elimination of IMD2 and suppression of IMD3 is reported in this research which is profoundly based on the integrated polarization multiplexing DPMZM with free dynamic range of $82 \mathrm{~dB}$. However, in this paper IMD2 has been completely eliminated whereas IMD3 has only been suppressed, while in our proposed structure all IMD's are completely eliminated.

In [18] is proposed and demonstrated a high linear analogue photonic link, based on a D-DPMZM with BPD. Moreover, third IMD3 and Second-Order Distortions (SOD) products have been completely eliminated. However, our system uses less RF shifters and it is much easier to be implement in practice as also shown in [19] whereas parts of the system has already been experimentally demonstrated.

\section{Mathematical model of the proposed system}

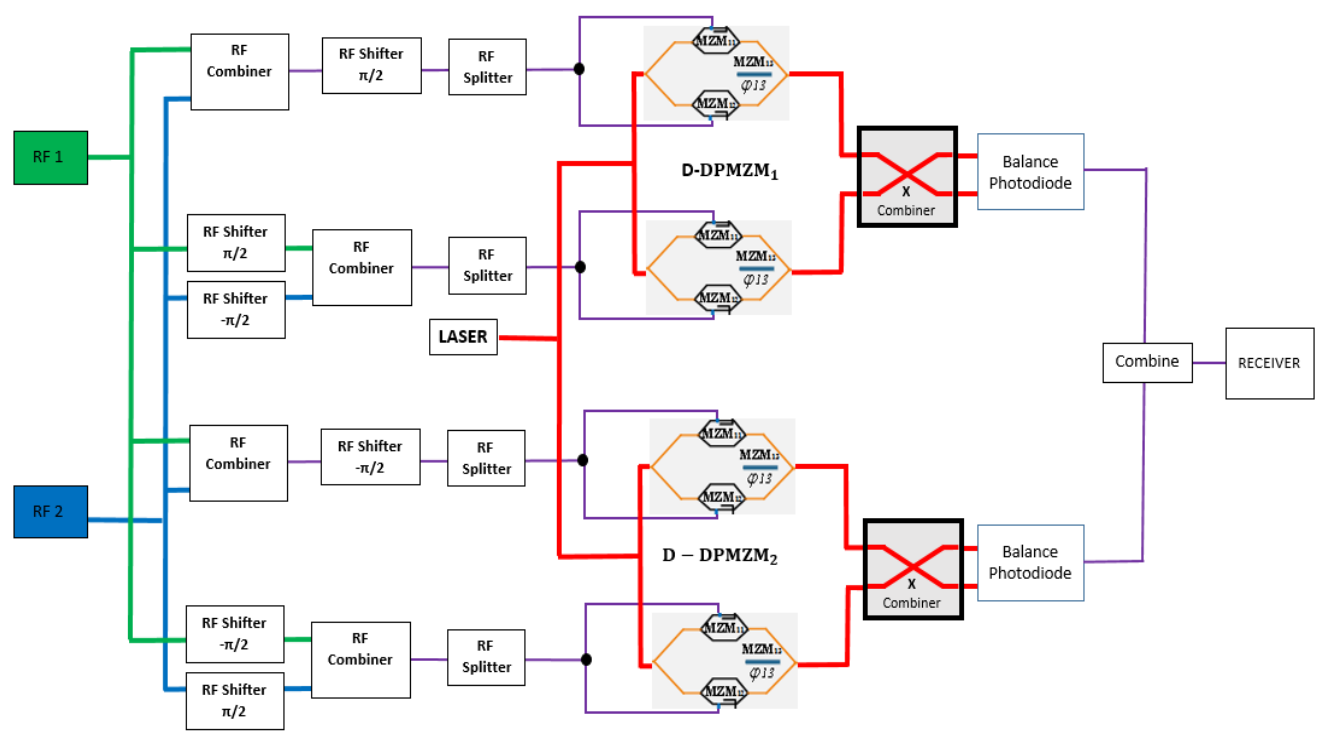

Fig. 1. Schematic diagram of the proposed AMPL system configuration using two D-DPMZM with two input frequencies.

The schematic diagram of proposed photonic link where all IMDs along Harmonics Distortions which are based on two D-DPMZM - have been eliminated by using balanced-photo-detectors, shown in the Fig. 1. In this configuration, we have used two-tone microwave frequency of 17 $\mathrm{GHz}$ and $17.5 \mathrm{GHz}$ using RF shifters by 90 degrees and 270 degrees, respectively. The two DDPMZM consist of two sub-dual electrode MZMs and two balance photo-detector combined by using a $3 \mathrm{~dB}$ power combiner.

Two RF signals are combined by RF combiner and then are shifted by 90 degrees for upper and lower arm of $\mathrm{MZM}_{1}\left(\mathrm{D}-\mathrm{DPMZM} \mathrm{M}_{1}\right)$ while frequency one is shifted by 270 degrees whereas frequency two is shifted by 90 degrees for upper and lower arm of MZM $_{2}$ (D-DPMZM 1 ). In D-DPMZM 2 the RF frequencies are combined and then shifted by 270 degrees for upper and lower arm of $\mathrm{MZM}_{1}\left(\mathrm{D}-\mathrm{DPMZM} \mathrm{M}_{2}\right.$ ), frequency one is shifted by 90 degrees and frequency two is shifted by 270 degrees for upper and lower arm of MZM $_{2}\left(D-D P M Z M_{2}\right)$. External DC bias is set to maximum for $\mathrm{MZM}_{1}$ and to quadrature for $\mathrm{MZM}_{2}$ for both D-DPMZMs. In addition, frequencies than are combined by $3 \mathrm{~dB}$ power combiner and detected by balanced photodetector. Results show that proposed configuration allows elimination of all IMDs and all even harmonic distortions.

The drive voltage with DC biases of the D-DPMZM ${ }_{1}$ for the schematic configuration illustrated in Fig. 1 can be expressed as:

$$
Q_{11}(t)=V_{m}\left\{\cos \left(\omega_{1} t+\frac{\pi}{2}\right)+\cos \left(\omega_{2} t+\frac{\pi}{2}\right)\right\}+V_{\pi}
$$




$$
\begin{gathered}
Q_{12}(t)=V_{m}\left\{\cos \left(\omega_{1} t+\frac{\pi}{2}\right)+\cos \left(\omega_{2} t+\frac{\pi}{2}\right)\right\} \\
Q_{21}(t)=V_{m}\left\{\cos \left(\omega_{1} t-\frac{\pi}{2}\right)+\cos \left(\omega_{2} t+\frac{\pi}{2}\right)\right\}+\frac{V_{\pi}}{2} \\
Q_{22}(t)=V_{m}\left\{\cos \left(\omega_{1} t-\frac{\pi}{2}\right)+\cos \left(\omega_{2} t+\frac{\pi}{2}\right)\right\}
\end{gathered}
$$

Where $Q_{11}(\mathrm{t})$ and $Q_{12}(\mathrm{t})$ are drive voltages on two electrodes of $\mathrm{MZM}_{1}\left(\mathrm{D}-\mathrm{DPMZM} \mathrm{M}_{1}\right.$ ); $Q_{21}(\mathrm{t})$ and $Q_{12}(\mathrm{t})$ are drive voltages on two electrodes of $\mathrm{MZM}_{2}\left(\mathrm{D}-\mathrm{DPMZM} \mathrm{M}_{1}\right) ; \mathrm{V}_{\mathrm{m}}$ represent the amplitude of the RF input signals. The laser power is expressed as: $E_{\mathrm{in}}(t)=E_{c} \mathrm{e}^{\mathrm{j} \omega_{\mathrm{c}} t}$ where the $E_{\mathrm{c}}$ is the input power and $\omega_{\mathrm{c}}$ is the angular frequency of the laser, consequently the output optical power in $\mathrm{MZM}_{1}\left(\mathrm{D}-\mathrm{DPMZM}_{1}\right)$ can be expressed as:

$$
E_{\text {out } 1 D-D P M Z_{1}}(t)=E_{\text {in }}(t)\left\{\exp \left(j \pi \frac{Q_{11}(t)}{V_{\pi}}\right)+\exp \left(-j \pi \frac{Q_{12}(t)}{V_{\pi}}\right)\right\}
$$

The output optical power in $\mathrm{MZM}_{2}\left(\mathrm{D}-\mathrm{DPMZM}_{1}\right)$ can be expressed as:

$$
E_{\text {out } 2 D-D P M Z_{1}}(t)=E_{\text {in }}(t)\left\{\exp \left(j \pi \frac{Q_{21}(t)}{V_{\pi}}\right)+\exp \left(-j \pi \frac{Q_{22}(t)}{V_{\pi}}\right)\right\}
$$

If $m=\frac{\pi V_{m}}{V_{\pi}}$ than by substituting equation (1) and (2) into equation (5) we obtain:

$$
E_{\text {out } 1 D-D P M Z_{1}}(t)=E_{\text {in }}(t)\left\{\begin{array}{l}
\exp \left(j m\left\{\sin \left(\omega_{1} t\right)+\sin \left(\omega_{2} t\right)\right\}+j \pi\right) \\
+\exp \left(-j m\left\{\sin \left(\omega_{1} t\right)+\sin \left(\omega_{2} t\right)\right\}\right)
\end{array}\right\}
$$

Applying a Jacobi-Anger Expansion in equation (7), we obtain:

$$
E_{\text {out } 2 D-D P M Z M_{1}}(t)=E_{\text {in }}(t) \sum_{n, m=-\infty}^{\infty} J_{n}(m) J_{m}(m) e^{j\left(n \omega_{1} t+\omega_{2} t\right)}\left[(-1)^{n+m} e^{j \pi}+1\right]
$$

Similarly, by substituting equation (3) and (4) into equation (6), we can derive the output optical power in $\mathrm{MZM}_{2}\left(\mathrm{D}-\mathrm{DPMZM}_{1}\right)$ :

$$
E_{\text {out } 2 D-D P M Z M_{1}(t)}=E_{\text {in }}(t)\left\{\begin{array}{l}
\exp \left(j m\left\{\sin \left(\omega_{1} t\right)-\sin \left(\omega_{2} t\right)\right\}\right)+j \frac{\pi}{2} \\
+\exp \left(-j m\left\{\sin \left(\omega_{1} t\right)-\sin \left(\omega_{2} t\right)\right\}\right)
\end{array}\right\}
$$

Applying a Jacobi-Anger Expansion in equation (9), we find:

$$
E_{\text {out } 2 D-D P M Z M_{1}}(t)=E_{\text {in }}(t) \sum_{n, m=-\infty}^{\infty} j_{n}(m) j_{m}(m) e^{j\left(n \omega_{1} t+\omega_{2} t\right)}\left[\begin{array}{l}
\left\{(-1)^{m}+1\right\} e^{j \frac{\pi}{2}} \\
+(-1)^{n}+1
\end{array}\right]
$$

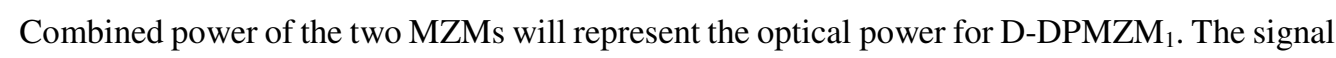
after $3 \mathrm{~dB}$ power combiner can be expressed as:

$$
\begin{aligned}
& E_{1 D-D P M Z M_{1}}(t)=\frac{E_{\text {out } 1 D-D P M Z M_{1}}(t)+E_{\text {out } 2 D-D P M Z M_{1}}(t)}{\sqrt{2}} \\
& E_{1 D-D P M Z M_{1}}(t)=\frac{E_{\text {out } 1 D-D P M Z M_{1}}(t)-E_{\text {out } 2 D-D P M Z M_{1}}(t)}{\sqrt{2}}
\end{aligned}
$$

The generated photocurrent $\mathrm{I}(t)$ after the balance-photodetector is: 


$$
I_{P D D-D P M Z M_{1}}(t)=R\left[\begin{array}{l}
E_{\text {out } 1 D-D P M Z M_{1}}(t) \cdot E_{\text {out } 1 D-D P M Z M_{1}}(t)^{*}- \\
E_{\text {out } 2 D-D P M Z M_{1}}(t) \cdot E_{\text {out } 2 D-D P M Z M_{1}}(t)^{*}
\end{array}\right]
$$

Where $\mathcal{R}$ is responsivity of the photodetector. By deploying Tayler series expansion to the third order in $m$, following expression can be derived.

$$
I_{P D D-D P M Z M_{1}}(t)=-\frac{1}{2} R P_{i n}\left\{\begin{array}{c}
8 m\left(\cos \left(\omega_{1} t\right)+\sin \left(\omega_{2} t\right)\right)+ \\
4 m^{2}\left(\cos \left(2 \omega_{1} t\right)-\cos \left(2 \omega_{2} t\right)\right) \\
+4 m^{3}\left(\begin{array}{c}
\sin \left(3 \omega_{1} t\right)+\sin \left(3 \omega_{2} t\right) \\
-\sin \left(\omega_{1} t\right)-\sin \left(\omega_{2} t\right)
\end{array}\right)
\end{array}\right\}+o(m)^{4}
$$

Similarly, we can derive equations for D-DPMZM 2 as follows:

$$
\begin{gathered}
Q_{11}(t)=V_{m}\left\{\cos \left(\omega_{1} t-\frac{\pi}{2}\right)+\cos \left(\omega_{2} t-\frac{\pi}{2}\right)\right\}+V_{\pi} \\
Q_{12}(t)=V_{m}\left\{\cos \left(\omega_{1} t+\frac{\pi}{2}\right)+\cos \left(\omega_{2} t+\frac{\pi}{2}\right)\right\} \\
Q_{21}(t)=V_{m}\left\{\cos \left(\omega_{1} t+\frac{\pi}{2}\right)+\cos \left(\omega_{2} t-\frac{\pi}{2}\right)\right\}+\frac{V_{\pi}}{2} \\
Q_{22}(t)=V_{m}\left\{\cos \left(\omega_{1} t+\frac{\pi}{2}\right)+\cos \left(\omega_{2} t-\frac{\pi}{2}\right)\right\}
\end{gathered}
$$

Where $Q_{11}(t)$ and $Q_{12}(t)$ are drive voltages on two electrodes of MZM $_{1}$ (D-DPMZM $)$; $Q_{21}(\mathrm{t})$ and $Q_{12}(\mathrm{t})$ are drive voltages on two electrodes of $\mathrm{MZM}_{2}(\mathrm{D}-\mathrm{DPMZM}) ; \mathrm{V}_{\mathrm{m}}$ represent the amplitude of the RF input signals. The laser power is expressed as: $E_{\text {in }}(t)=E_{\mathrm{c}} \mathrm{j}^{\mathrm{j} \omega_{\mathrm{c}} \mathrm{t}}$ where the $E_{c}$ is the input power and $\omega_{c}$ is the angular frequency of the laser, consequently the output optical in $\mathrm{MZM}_{1}\left(\mathrm{D}-\mathrm{DPMZM}_{2}\right)$ can be expressed as:

$$
E_{\text {out } 1 D-D P M Z 2}(t)=E_{\text {in }}(t)\left\{\exp \left(j \pi \frac{Q_{11}(t)}{V_{\pi}}\right)+\exp \left(-j \pi \frac{Q_{12}(t)}{V_{\pi}}\right)\right\}
$$

And the output optical power in $\mathrm{MZM}_{2}\left(\mathrm{D}-\mathrm{DPMZM}_{2}\right)$ can be expressed as:

$$
E_{\text {out } 2 D-D P M Z_{2}}(t)=E_{\text {in }}(t)\left\{\exp \left(j \pi \frac{Q_{21}(t)}{V_{\pi}}\right)+\exp \left(-j \pi \frac{Q_{22}(t)}{V_{\pi}}\right)\right\}
$$

If $m=\frac{\pi V_{m}}{V_{\pi}}$ than by substituting equation (15) and (16) into equation (19), we obtain:

$$
E_{\text {out } 1 D-D P M Z 2}(t)=E_{\text {in }}(t)\left\{\begin{array}{l}
\exp \left(j m\left\{\sin \left(\omega_{1} t\right)+\sin \left(\omega_{2} t\right)\right\}+j \pi\right) \\
+\exp \left(j m\left\{\sin \left(\omega_{1} t\right)+\sin \left(\omega_{2} t\right)\right\}\right)
\end{array}\right\}
$$

Applying a Jacobi-Anger Expansion in equation 20 we get:

$$
E_{\text {out } 1 D-D P M Z M 2}(t)=E_{\text {in }}(t) \sum_{n, m=-\infty}^{\infty} J_{n}(m) J_{m}(m) e^{j\left(n \omega_{1} t+\omega_{2} t\right)}\left[(-1)^{n+m} e^{j \pi}+1\right]
$$

Similarly, by substituting equation (17) and (18) into equation (20), we can derive the output optical power in $\mathrm{MZM}_{2}\left(\mathrm{D}-\mathrm{DPMZM}_{2}\right)$ :

$$
E_{\text {out } 2 D-D P M Z M_{2}(t)}=E_{\text {in }}(t)\left\{\begin{array}{l}
\exp \left(j m\left\{-\sin \left(\omega_{1} t\right)+\sin \left(\omega_{2} t\right)\right\}\right)+j \frac{\pi}{2} \\
+\exp \left(-j m\left\{-\sin \left(\omega_{1} t\right)+\sin \left(\omega_{2} t\right)\right\}\right)
\end{array}\right\}
$$

Applying a Jacobi-Anger Expansion in equation (22), we obtain: 
$E_{\text {out } 2 D-D P M Z M 2}(t)=E_{\text {in }}(t) \sum_{n, m=-\infty}^{\infty} j_{n}(m) j_{m}(m) e^{j\left(n \omega_{1} t+\omega_{2} t\right)}\left[\begin{array}{l}\left\{(-1)^{m}+1\right\} e^{j \frac{\pi}{2}} \\ +(-1)^{n}+1\end{array}\right]$

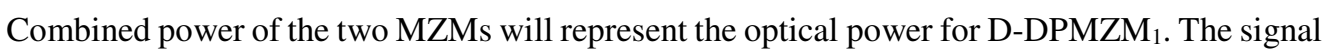
after $3 \mathrm{~dB}$ power combiner can be expressed as:

$$
\begin{aligned}
& E_{2 D-D P M Z M_{2}}(t)=\frac{E_{\text {out } 1 D-D P M Z M_{2}}(t)+E_{\text {out } 2 D-D P M Z M 2}(t)}{\sqrt{2}} \\
& E_{2 D-D P M Z M 2}(t)=\frac{E_{\text {out } 1 D-D P M Z M 2}(t)-E_{\text {out } 2 D-D P M Z M_{2}}(t)}{\sqrt{2}}
\end{aligned}
$$

The generated photocurrent $\mathrm{I}_{\mathrm{PD}_{-} \mathrm{D}-\mathrm{DPMZM}}(\mathrm{t})$ after the balance-photodetector is:

$$
I_{P D D-D P M Z M 2}(t)=R\left[\begin{array}{l}
E_{\text {out } 1 D-D P M Z M_{2}}(t) \cdot E_{\text {out } 1 D-D P M Z M_{2}}(t)^{*}- \\
E_{\text {out } 2 D-D P M Z M 2}(t) \cdot E_{\text {out } 2 D-D P M Z M 2}(t)^{*}
\end{array}\right]
$$

Where $\mathcal{R}$ is responsivity of photodetector. By using Tayler series expansion to the third order in $\mathrm{m}$, following expression can be derived:

$$
I_{P D D-D P M Z M 2}(t)=-\frac{1}{2} R P_{i n}\left\{\begin{array}{c}
8 m\left(\cos \left(\omega_{1} t\right)+\sin \left(\omega_{2} t\right)\right)+ \\
-4 m^{2}\left(\cos \left(2 \omega_{1} t\right)-\cos \left(2 \omega_{2} t\right)\right) \\
+4 m^{3}\left(\begin{array}{c}
\sin \left(3 \omega_{1} t\right)+\sin \left(3 \omega_{2} t\right) \\
-\sin \left(\omega_{1} t\right)-\sin \left(\omega_{2} t\right)
\end{array}\right)
\end{array}\right\}+o(m)^{4}
$$

Combining power after two balance-photodetectors

$$
I_{P D}(t)=I_{P D D-D P M Z M 1}(t)+I_{P D D-D P M Z M 2}(t)
$$

Then we obtain:

$$
I_{P D}(t)=R P_{i n}\left\{\begin{array}{l}
16 m\left(\cos \left(\omega_{1} t\right)+\sin \left(\omega_{2} t\right)\right)+ \\
+8 m^{3}\left(\begin{array}{c}
\sin \left(3 \omega_{1} t\right)+\sin \left(3 \omega_{2} t\right) \\
-\sin \left(\omega_{1} t\right)-\sin \left(\omega_{2} t\right)
\end{array}\right)
\end{array}\right\}+o(m)^{4}
$$

From results in equation (28), it can be seen that the third order Intermodulation Distortion of frequency $2 \omega_{2}-\omega_{1}$ and $2 \omega_{1}-\omega_{2}$ is eliminated. Second order harmonic and intermodulation of frequency is $\omega_{2}-\omega_{1}, \omega_{1}-\omega_{2}$, and $2 \omega_{2}-2 \omega_{1}, 2 \omega_{1}-2 \omega_{2}$. We have used the Tayler series to higher order (up to ninth order) and to all intermodulation distortions and even harmonic distortions does not exist which means that the modulation index increases the IMDs and even harmonic distortions will not exist in this model.

\section{Simulation results and discussions}

Simulation results are undertaken based on the developed mathematical model representing the novel RF system configuration Fig. 1. As stated above, the schematic configuration of proposed microwave photonic signal linearization includes laser, two tone microwave frequencies, six shifters four of which are 90-degrees and two 270-degree, $3 \mathrm{~dB}$ power combiner, two DDPMZM and two balance-photodetector. Laser optical power of $20 \mathrm{dBm}$ is used, and two signals with frequency of $17 \mathrm{GHz}$ and $17.5 \mathrm{GHz}$ are investigated. The input laser light is split in four equal paths and modulated at four sub-MZM $\mathrm{s}$.

In D-DPMZM 1 lower and upper branch of $\mathrm{MZM}_{1}$ the RF signal $\omega_{1}$ and $\omega_{2}$ are shifted by 90 degrees, while in $M_{2 M}$ RF signal $\omega_{1}$ is shifted by $\frac{\pi}{2}$ and $\omega_{2}$ is shifted by $-\frac{\pi}{2}$, frequencies are than combined by RF combiner and modulated in upper and lower branch. Output modulated 
signal from $\mathrm{MZM}_{1}$ and $\mathrm{MZM}_{2}$ are combined by $3 \mathrm{~dB}$ power combiner than detected by balanced-photodetector. In D-DPMZM 2 RF signal are shifted and combined same as in DDPMZ1 but with opposite phase, output modulated signal from $\mathrm{MZM}_{1}$ and $\mathrm{MZM}_{2}$ are combined and detected by balanced-photodetector two. Electrical signal from both balancedphotodetector is combined, obtained results are shown in Fig. 2.
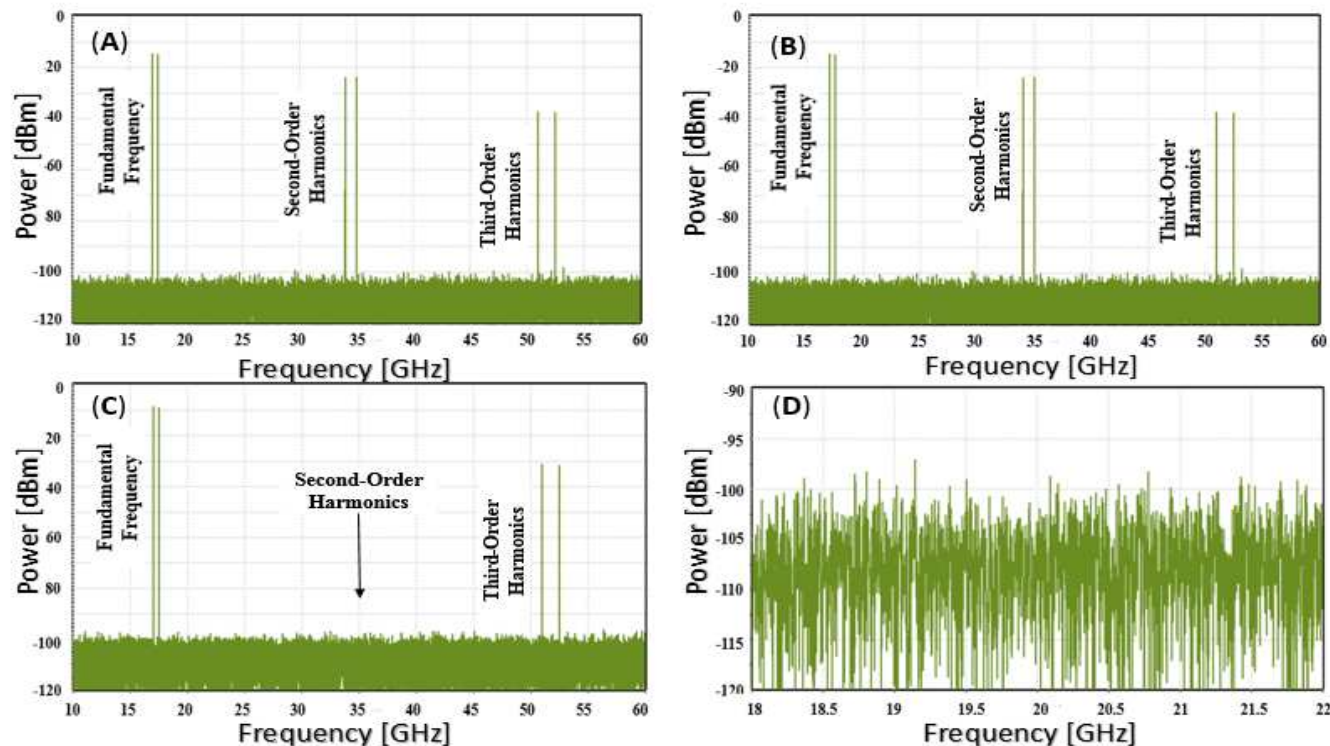

Fig. 2. Electrical spectrum of the proposed structure; (A) Two single subcarriers from D-DPMZM1 without any intermodulation distortions, (B) Two single subcarriers from D-DPMZM2 without any intermodulation distortions, (C) Two single subcarriers after combining without any SOH, (D) shows the non-existence of IMD2 and SOH

Fig. 2(A) shows signal output from D-DPMZM $\mathrm{SOH}$ is present as shown equation (14). Similarly, Fig. 2(B) illustrates the output from D$\mathrm{DMZM}_{2}$ with opposite SOH field shown in equation (27) which enables the elimination of SOH. Therefore, SFDR in this system is limited only by Third Order Harmonics (TOH). The TOH are quite far from fundamental signal and can be easily filter by using low power electrical filter.

By comparing our obtained results with our previous experimental results of similar RF system publish recently in [18], it can be confirmed that we have managed to improve a signal linearization significantly. In the published paper [18], we have demonstrated suppression of IMDs and SOH experimentally and benchmarked by the developed mathematic model, however in [18] only IMD's and SOH are suppressed, shown in equation (14) section 2 Mathematic Modelling, where in this proposed RF system configuration, we have eliminated SOH Fig. 2 (D) and all other IMD's Fig. 2 (C). Obtained simulations shown in Fig.2 (C) have been backed up by the developed mathematical modelling equation (28), showing Tayler series up to forth order which confirms that the IMD's and SOH do not exist. In order to verify simulation results, we have used Tayler series up to $9^{\text {th }}$ order and still evidence the clear the match.
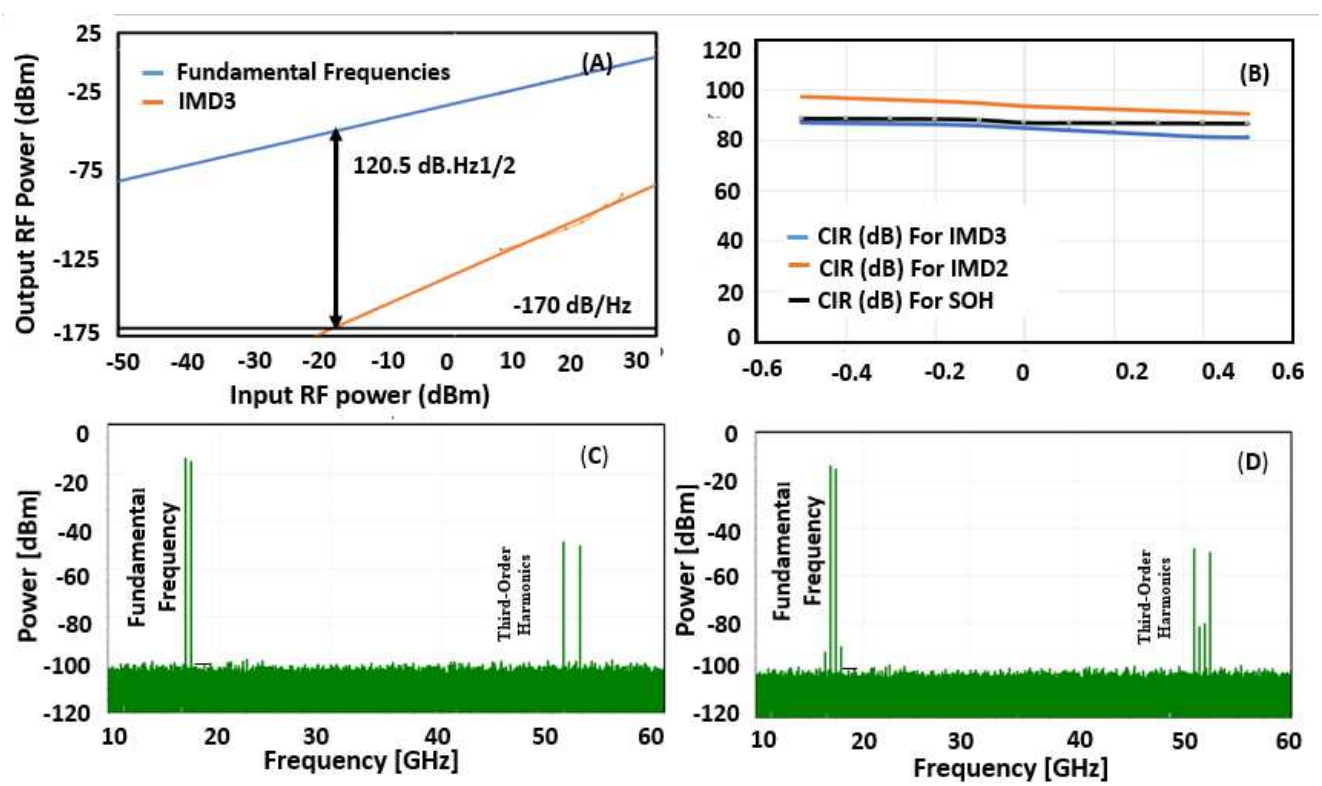

Fig. 3. (A) SFDR performance of proposed AMPL with Dual Parallel MZMs, (B) System stability analyses (C). Two single subcarriers after changing RF shifters for 1 degree, (D). Two single subcarriers after changing RF shifters for 1 degree and adding fiber between BPDs and 3dB power combiner. 


\begin{tabular}{|c|c|c|c|c|}
\hline & $\mathrm{MZM}_{1}$ & $\mathrm{MZM}_{2}$ & $\mathrm{MZM}_{3}$ & $\mathrm{MZM}_{4}$ \\
\hline VpiDC/VpiRf & $3.5 \mathrm{~V}$ & $3.5 \mathrm{~V}$ & $3.5 \mathrm{~V}$ & $3.5 \mathrm{~V}$ \\
\hline Insertion Loss & $6 \mathrm{~dB}$ & $6 \mathrm{~dB}$ & $6 \mathrm{~dB}$ & $6 \mathrm{~dB}$ \\
\hline Extinction Ratio & $21.8 \mathrm{~dB}$ & $18.5 \mathrm{~dB}$ & $21.8 \mathrm{~dB}$ & $18.5 \mathrm{~dB}$ \\
\hline dVpiDC_dTemperature & $0.005 \mathrm{~V} / \mathrm{deg} \mathrm{C}$ & $0.005 \mathrm{~V} / \mathrm{deg} \mathrm{C}$ & $0.005 \mathrm{~V} / \mathrm{deg} \mathrm{C}$ & $0.005 \mathrm{~V} / \mathrm{deg} \mathrm{C}$ \\
\hline dVpiRF_dTemperature & $0.0015 \mathrm{~V} / \mathrm{deg} \mathrm{C}$ & $0.0015 \mathrm{~V} / \mathrm{deg} \mathrm{C}$ & $0.0015 \mathrm{~V} / \mathrm{degC}$ & $0.0015 \mathrm{~V} / \mathrm{deg} \mathrm{C}$ \\
\hline OperatingTemperature & $25 \operatorname{deg} \mathrm{C}$ & $25 \operatorname{deg} \mathrm{C}$ & $25 \operatorname{deg} \mathrm{C}$ & $25 \operatorname{deg} \mathrm{C}$ \\
\hline ReferenceTemperature & $25 \operatorname{deg} C$ & $25 \operatorname{deg} C$ & $25 \operatorname{deg} C$ & $25 \operatorname{deg} C$ \\
\hline ElectrodeLengthUpper & $23 \mathrm{~mm}$ & $23 \mathrm{~mm}$ & $23 \mathrm{~mm}$ & $23 \mathrm{~mm}$ \\
\hline IndexMismatchUpper & 0.05 & 0.05 & 0.05 & 0.05 \\
\hline MicrowaveLossUpper & $0.002 \mathrm{~dB} / \mathrm{m} / \mathrm{sqrt}(\mathrm{Hz})$ & $0.002 \mathrm{~dB} / \mathrm{m} / \mathrm{sqrt}(\mathrm{Hz})$ & $0.002 \mathrm{~dB} / \mathrm{m} / \mathrm{sqrt}(\mathrm{Hz})$ & $0.002 \mathrm{~dB} / \mathrm{m} / \mathrm{sqrt}(\mathrm{Hz})$ \\
\hline ElectrodeLengthLower & $23 \mathrm{~mm}$ & $23 \mathrm{~mm}$ & $23 \mathrm{~mm}$ & $23 \mathrm{~mm}$ \\
\hline IndexMismatchLower & 0.05 & 0.05 & 0.05 & 0.05 \\
\hline MicrowaveLossLower & $0.002 \mathrm{~dB} / \mathrm{m} / \mathrm{sqrt}(\mathrm{Hz})$ & $0.002 \mathrm{~dB} / \mathrm{m} / \mathrm{sqrt}(\mathrm{Hz})$ & $0.002 \mathrm{~dB} / \mathrm{m} / \mathrm{sqrt}(\mathrm{Hz})$ & $0.002 \mathrm{~dB} / \mathrm{m} / \mathrm{sqrt}(\mathrm{Hz})$ \\
\hline
\end{tabular}

Table.1 MZM Parameters used in our simulation

In the Fig. 3 (A), we present the SFDR performance analysis of the proposed system. As we have mathematically presented, the proposed system in an ideal condition, it will not produce any IMD3, IMD2 or SOH, however in real life it is almost impossible to have an ideal condition, therefore we have changed the modulator parameter to match with our practical laboratory components (shown in Table 1); modulators and RF shifters for 1 degree to measure the SFDR3. Fig. 3 (A) shows SFDR3 for the proposed link is $120.5 \mathrm{~dB} \cdot \mathrm{Hz}^{1 / 2}$ from -170 noise floor.

To demonstrate the performance of the proposed RF system configuration with reference to the bias drift, we have investigated the following CNR for IMD3, IMD2 and SOH, shown in Fig. 3(B). As it can be seen from results, the CIR for IMD3, IMD2 and SOH are almost constant at a bias drift of up to $\pm 0.5 \mathrm{~V}$, therefore system is stable. Due to fact that IMDs and $\mathrm{SOH}$ are eliminated, the propose system performance is stable as the operating points voltage changes.

Next, we have tested the system purity by varying the parameters of the modulator (shown in Table. 1) to match with practical modulators and by changing RF shifters for one degree. As can be seen, the obtained results are similar to those in Fig. 3(C). Furthermore, by using the same parameters for modulators and RF shifters, we have tasted the system further by adding different fiber lengths between $3 \mathrm{~dB}$ combiner and BPDs (20-meter fiber between $3 \mathrm{~dB}$ combiner and BPD1 and 19-meter fiber between $3 \mathrm{~dB}$ combiner and BPD2) results are shown in Fig. 3(D). Simulation from Fig. 3(D) shows confirms that there is no SOH, however, if the length of fiber optic cable is not the same, then IMD3 starts coming up the noise floor.
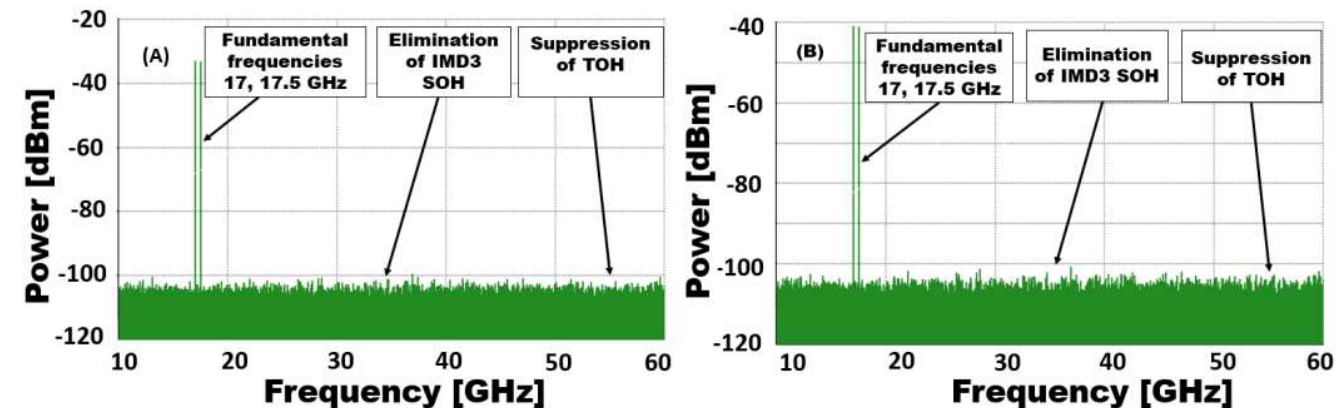

Fig. 4. (A) Two single subcarriers after including $42 \mathrm{~dB}$ losses, (B) Two single subcarriers after including $42 \mathrm{~dB}$ losses and changing the input of laser to $15 \mathrm{~dB}$.

The proposed model consists of 6 RF shifters, 4 splitters and 4 combiners, therefore, it known there will be losses. In this regard, in the simulation model we have included all potential losses which might occur in practice. Attenuates on each connection have been considered in order to present the losses for each shifter, splitter and combiner, obtained result are shown in Fig. 4(A). Losses added to the system is $42 \mathrm{~dB}$, hence the dynamic range is not as shown in an ideal case in Fig 2, however, the obtained results demonstrate that the dynamic range has not been decreased in regard to $\mathrm{TOH}$ and $\mathrm{TOH}$ which have been suppressed under the noise floor. Furthermore, the system performance has been tested by changing the input of the laser to 15 $\mathrm{dB}$, which includes losses as mention above in order to investigate the effects on SFDR, obtained result are shown in Fig. 4(B). 


\section{Conclusion}

In this paper, we have developed and demonstrated a high linear analogue photonic link where all IMDs and $\mathrm{SOH}$ have been eliminated completely, by deploying unique system architecture which consisting of 6 RF shifters, 2 D-DPMZMs and 2 balance photodetectors. The proposed analog photonic link configuration which exhibits significant performance is easy to implement in practice [18]. We have demonstrated that IMDs and SOH distortion products can be completely eliminated, which is a challenging task to achieve due to the fact that if signals are very close to each other, it will be very difficult to filter $\mathrm{SOH}$ by using various filtering techniques. We have demonstrated that the system SFDR is only limited by TOH distortions which are far apart from fundamental signal and can easily be filter by using low electronic filters. We also have benchmarked the developed mathematical model and system simulations, and it has been confirmed that the mathematical model and the simulation results match very well, confirming that the proposed system can be implemented in practise. Additionally, we have also demonstrated the SFDR of $120.5 \mathrm{~dB} \cdot \mathrm{Hz}^{1 / 2}$. 


\section{References}

1. A.J. Cooper, Electron. Lett. 26 (24) (1990) 2054

2. B. M. Haas and T. E. Murphy: A Simple, Linearized, Phase-Modulated Analog Optical Transmission System. In IEEE Photonics Technology Letters, vol. 19, no. 10, pp. $729-731$ (2007).

3. B. Masella, B. Hraimel and X. Zhang: Enhanced Spurious-Free Dynamic Range Using Mixed Polarization in Optical Single Sideband Mach-Zehnder Modulator. In Journal of Lightwave Technology, vol. 27, no. 15, pp. 3034-3041 (2009).

4. C. Cox, Analog Optical Links, Theory and Practice, Cambridge University, (2004)

5. Dan Zhu, Jian Chen, and Shilong Pan: Multi-octave linearized analog photonic link based on a polarizationmultiplexing dual-parallel Mach-Zehnder modulator. Opt. Express 24, 11009-11016 (2016).

6. E. Xu, M. Zhang, P. Li, and Z. Zhang: Dynamic-Range Enhancement in Microwave Photonic Link Based on Single-Sideband Phase Modulation. In Asia Communications and Photonics Conference 2016, OSA Technical Digest (online) (Optical Society of America, 2016), paper AF2A.16.

7. F. Pail; S. Haxha, T. N. Mirza, M. S. Alom: Microwave Photonic Down-conversion with Improved Conversion Efficiency and SFDR. In IEEE Access, 6, 8089-8097 (2018).

8. J. Yao, Microwave photonics, J. Lightw. Tcchnol. 27 (3) 225-314, (2009).

9. Jean-Marc Mérolla, Yuri Mazurenko, Jean-Pierre Goedgebuer, Henri Porte, and William T. Rhodes: Phasemodulation transmission system for quantum cryptography. Opt. Lett. 24, 104-106 (1999)

10. Menghao Huang, Jianbin Fu, and Shilong Pan: Linearized analog photonic links based on a dual-parallel polarization modulator. Opt. Lett. 37, 1823-1825 (2012)

11. Shemsi Shaqiri, Shyqyri Haxha, and Taimur N. Mirza: Elimination of odd and even intermodulation distortions of analog microwave photonics link based on GaAs MZMs. Opt. Express 28, 17521-17531 (2020).

12. Shemsi Shaqiri, Shyqyri Haxha. Linearization and down-conversion of microwave photonics Signal based on dual-drive dual-parallel mach-zehnder modulator with eliminated 3rd intermodulation and 2nd distortions. Elsevir, Vol.204, pp. 164103 (2020).

13. T. Jiang, R. Wu, S. Yu, D. Wang and W. Gu: A Novel High-Linearity Microwave Photonic Link Based on the Strategy of Adding a Compensation Path Using a Bidirectional Phase Modulator. In IEEE Photonics Journal, vol. 8 , no. 5, pp. 1-7 (2016).

14. T. N. Mirza, S. Haxha and I. Dayoub: A Linearized Analog Microwave Photonic Link with an Eliminated EvenOrder Distortions. In IEEE Systems Journal (DOI: 10.1109/JSYST.2021.3051394), pp.1-9 (2021).

15. W. Jiang Et Al: A Linearization Analog Photonic Link with High Third-Order Intermodulation Distortion Suppression Based on Dual-Parallel Mach-Zehnder Modulator. In IEEE Photonics J., vol. 7, no. 3, pp. 1-8, (2015)

16. Weize Qin and Wei Jiang: The performance analysis of microwave photonic frequency conversion using doublesideband suppressed-carrier and balance detection. IEEE International Conference on Communication ProblemSolving (ICCP), Guilin, pp. 582-585 (2015)

17. Wu Zhang, Aijun Wen, Xinyao Xu, Weile Zhai, Kongkun Wei, Huixing Zhang: Dual-wavelength linearization of analog photonic link based on PM-IM conversion. Optics Communications, Vol. 4, pp.20174-178 (2018).

18. X. Zou, B. Lu, W. Pan, L. Yan, A. Stohr, J. Yao, Photonics for microwave measurements, Laser Photonics Rev. 10 (5) (2016) 711-734.

19. Z. Xie, S. Yu, S. Cai and W. Gu: Linearized Phase-Modulated Analog Photonic Link with Large Spurious-Free Dynamic Range. Asia Communications and Photonics Conference (ACP), Wuhan, China, pp. 1-3 (2016). 
Figures

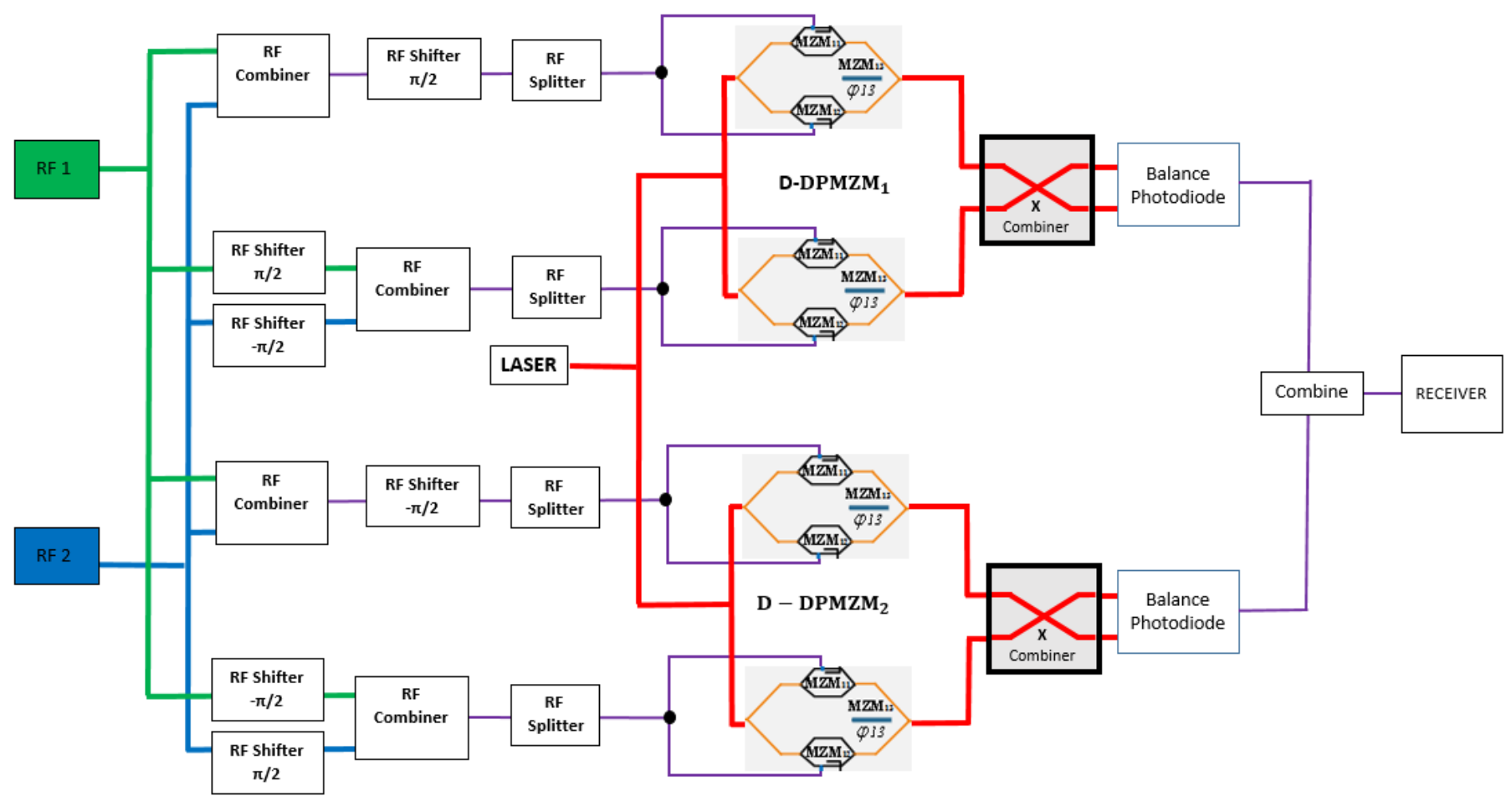

\section{Figure 1}

Schematic diagram of the proposed AMPL system configuration using two D-DPMZM with two input frequencies. 

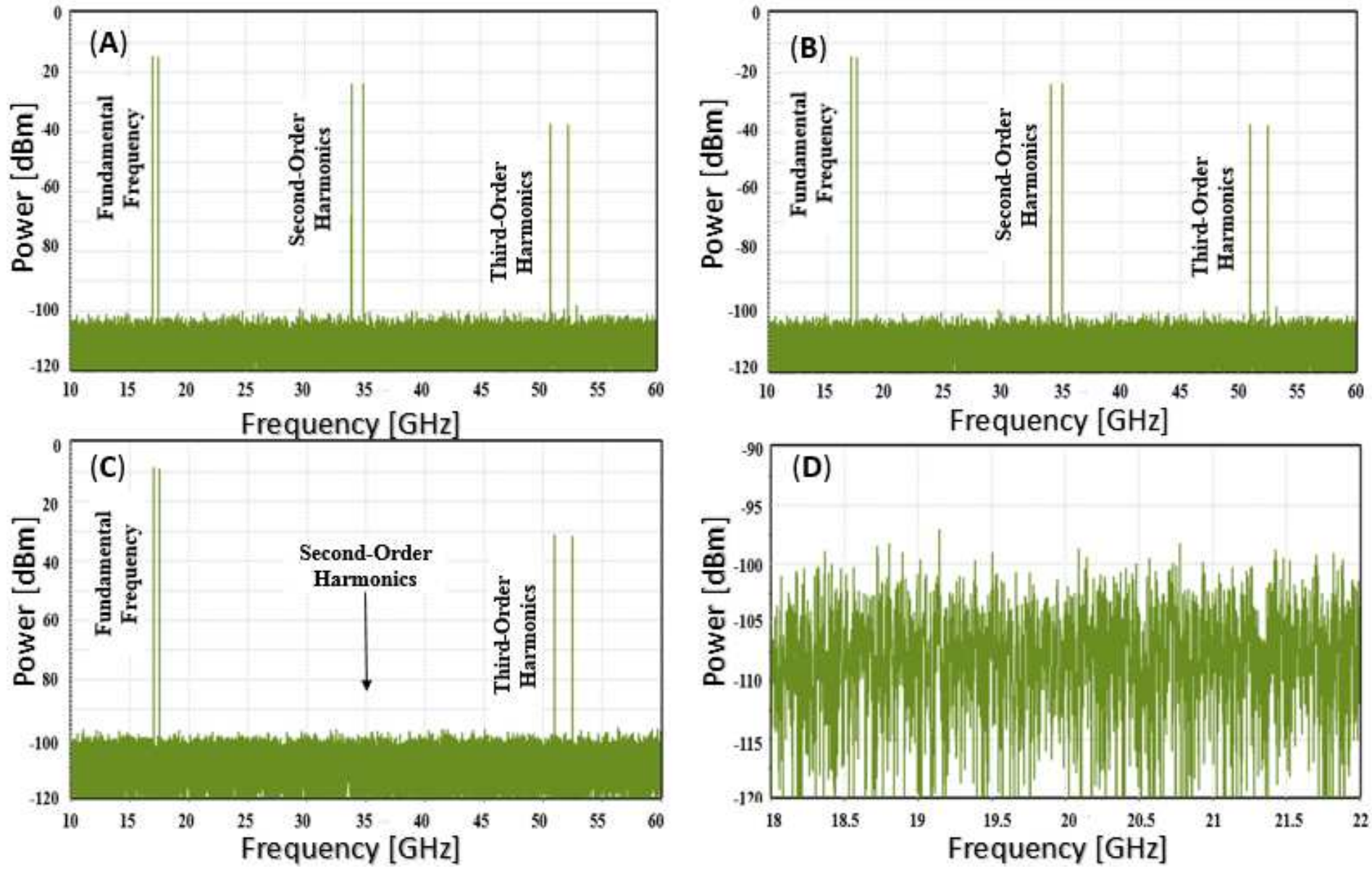

Figure 2

Electrical spectrum of the proposed structure; (A) Two single subcarriers from D-DPMZM1 without any intermodulation distortions, (B) Two single subcarriers from D-DPMZM2 without any intermodulation distortions, (C) Two single subcarriers after combining without any $\mathrm{SOH}$, (D) shows the non-existence of IMD2 and SOH 

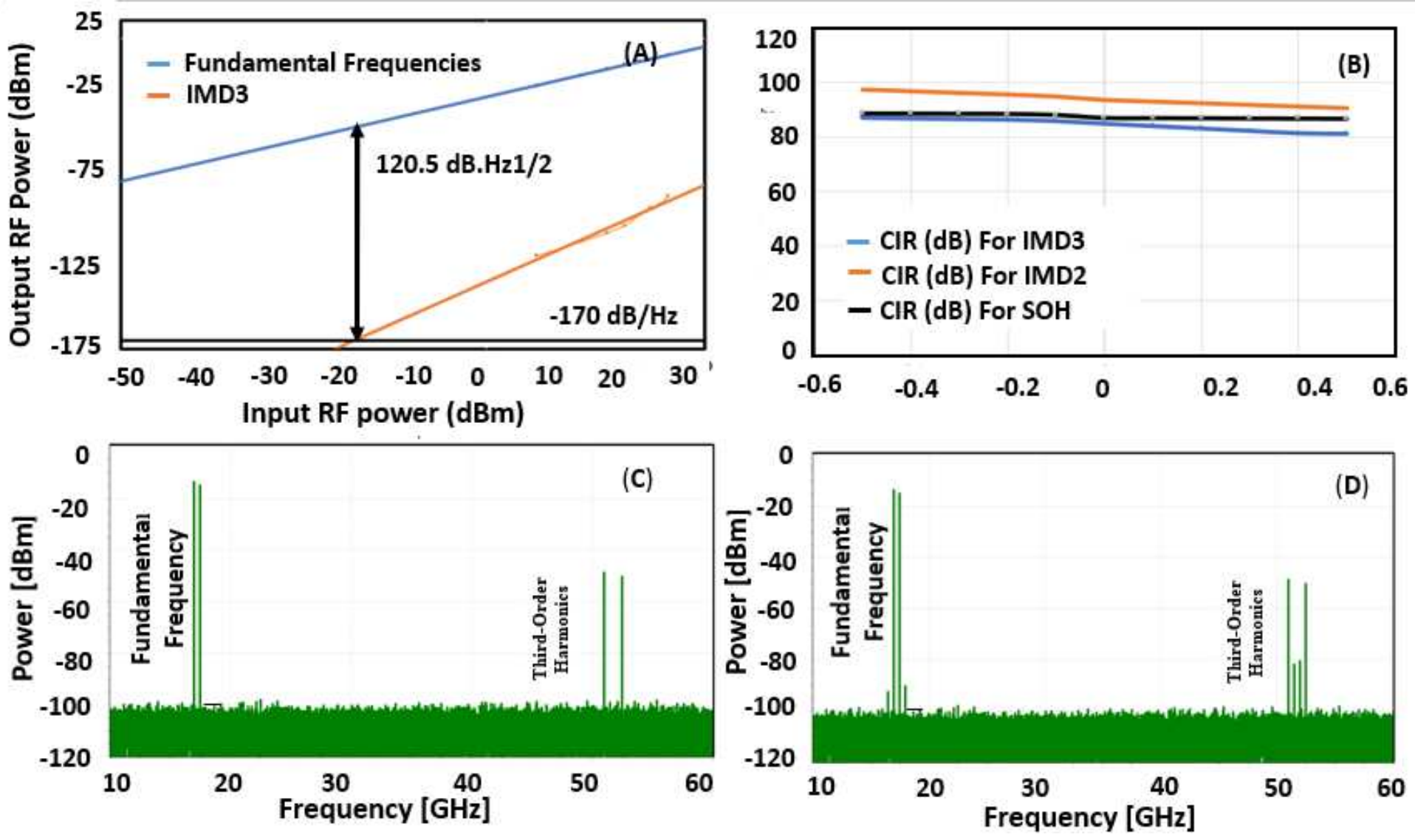

Figure 3

(A) SFDR performance of proposed AMPL with Dual Parallel MZMs, (B) System stability analyses (C). Two single subcarriers after changing RF shifters for 1 degree, (D). Two single subcarriers after changing RF shifters for 1 degree and adding fiber between BPDs and 3dB power combiner.
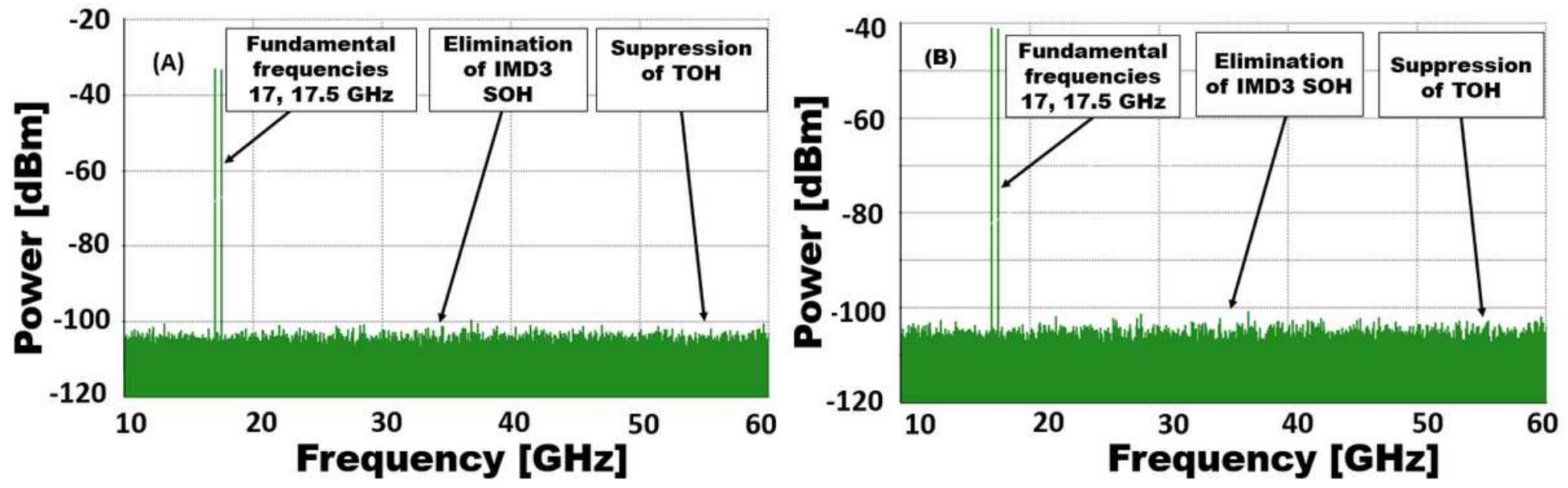

Figure 4

(A) Two single subcarriers after including $42 \mathrm{~dB}$ losses, (B) Two single subcarriers after including $42 \mathrm{~dB}$ losses and changing the input of laser to $15 \mathrm{~dB}$. 